\title{
Modulations physiologiques et immunologiques du diabète gestationnel et de la macrosomie : implication de la programmation in utero
}

\author{
Jean-Marc ATEGBO ${ }^{1 *}$, Tèniola Isabelle SACRAMENTO ${ }^{1}$, Laye Karim DRAMANE ${ }^{1}$ \\ et Akhtar NAIM KHAN ${ }^{2}$ \\ ${ }^{1}$ Faculté des Sciences et Techniques, Université d'Abomey-Calavi, BP 526 Cotonou, Bénin. \\ ${ }^{2}$ Université de Bourgogne, UPRES EA 4183 "Lipides \& Signalisation Cellulaire”, Faculté des Sciences de la \\ Vie, 6 Bd Gabriel, 21000 Dijon, France. \\ *Auteur correspondant, E-mail : jmarcategbo@yahoo.fr, marc.ategbo@fast.uac.bj
}

\section{RESUME}

Le diabète gestationnel aboutit fréquemment à la macrosomie et à l'obésité foetale. Il semble que les anomalies du métabolisme des carbohydrates et des lipides, observées chez les bébés macrosomiques nés de mères diabétiques, sont dues à l'hyperglycémie maternelle qui induit l'hyperinsulinémie fotale. Nous avons développé un modèle animal de macrosomie et avons évalué l'incidence de l'obésité chez ces animaux. Les bébés macrosomiques, issus de mères diabétiques, sont prédisposés à développer, avec l'âge, l'intolérance au glucose, l'insulino-résistance et l'obésité. En outre, les cellules-T des rattes diabétiques et de leurs nouveau-nés macrosomiques semblent présenter un défaut dans la transduction du signal. Il semble que la programmation in utero au cours du diabète gestationnel crée une "mémoire métabolique" qui est responsable du développement de l'obésité chez les nouveau-nés macrosomiques et de l'apparition de maladies métaboliques à l'âge adulte.

(C) 2010 International Formulae Group. All rights reserved.

Mots clés : Diabète gestationnel, macrosomie, métabolisme glucidique, métabolisme lipidique, mémoire métabolique.

\section{INTRODUCTION}

Le Diabète gestationnel (DG) est le plus fréquent désordre métabolique de la grossesse qui affecte $1-10 \%$ de toutes les grossesses (Gabbe, 1986). C'est généralement un phénomène temporaire qui apparaît pendant la grossesse, habituellement au second ou au troisième trimestre. Cependant, il pourrait causer des complications métaboliques non seulement à la mère mais aussi à l'enfant. Les complications majeures $\mathrm{du}$ diabète gestationnel comportent les désordres hypertensifs maternels, l'hypoglycémie néonatale, la jaunisse (ictère), et les traumatismes à la naissance incluant la dystocie des épaules (Coustan, 1997). L'effet le plus communément rapporté sur les nouveau-nés est la macrosomie ("gros bébés"), habituellement définie soit comme un poids à la naissance supérieur à $4 \mathrm{~kg}$ ou audessus du $90^{\text {th }}$ percentile pour l'âge gestationnel. L'incidence de la macrosomie avoisine $50 \%$ en cas de diabète de type 2 (Evers et al., 2002) et elle varie entre 18 et $30 \%$ dans les autres cas de diabète (Jensen et al., 2003).

Il y a une probabilité de $40-60 \%$ pour un enfant obèse de demeurer tel jusqu'à l'âge 
adulte (Basdevant, 1998). Désormais, la macrosomie constitue l'un des facteurs qui pourrait contribuer à l'émergence de l'obésité infantile. En général, la prévalence de l'obésité infantile en France, parmi les enfants âgés de 4 à 8 ans, a été de 10-14\% (Tauber et al., 1998).

Les enfants macrosomiques, nés de mères diabétiques, sont aussi exposés à la mort fœetale, la prématurité, les traumatismes à la naissance, les syndromes de détresse respiratoire et plus fréquemment, ces bébés présentent un risque élevé de devenir obèse, une diminution de la tolérance au glucose et un diabète de type 2 à l'âge adulte (Cox, 1994). L'hyperglycémie maternelle induit plusieurs altérations dans le métabolisme des glucides et des lipides conduisant à l'hyperglycémie foetale et à l'hyperinsulinémie (Ornoy et al., 1999). L'influence $\mathrm{du}$ diabète gestationnel sur l'incidence de la macrosomie chez la ratte a été étudiée par plusieurs équipes de recherche (Khan et Merzouk, 2003; Yessoufou et al., 2006). Ces animaux macrosomiques, nés de mères diabétiques, étaient devenus non seulement obèses à l'âge adulte mais aussi, avaient développé le diabète de type 2, associé à une hyperglycémie et une hyperinsulinémie avec des taux élevés de lipoprotéines et de VLDL (Souliman-Moukhtari et al., 2005). Les anomalies du métabolisme des lipides (augmentations du cholestérol total, LCAT, LPL, HTGL, ACAT, HMG CoA reductase et $7 \alpha$-hydroxylase, LPL dans le foie) ont été également mises en évidence chez ces animaux (Khan et Merzouk, 2003).

Puisque les complications dues au diabète gestationnel peuvent apparaître chez la mère et les nouveau-nés, une gestion efficiente de cette pathologie est obligatoire.

\section{Le stress oxydatif est impliqué dans le diabète gestationnel et la macrosomie}

Les taux élevés du glucose sanguin chez les mères diabétiques et leurs nouveaunés ont été considérés comme induisant le stress oxydatif (Kamath et al., 1998) qui, à son tour, provoque la génération de radicaux libres
(ROS), toxiques pour les cellules, en particulier les membranes plasmiques où ils interagissent avec la membrane lipidique. Au cours du diabète aussi bien que de la macrosomie, la glycation protéique et l'autooxydation glucidique peuvent générer des radicaux libres, qui à leur tour catalysent la peroxydation lipidique (Hunt et al., 1990). Les effets biologiques de ces ROS sont normalement contrôlés in vivo par des antioxydants endogènes tels les vitamines $\mathrm{A}$, $\mathrm{C}$ et $\mathrm{E}$, le glutathion et les enzymes antioxydants (Guemouri et al., 1991 ; Therond et al., 2000). Ainsi, le traitement par des antioxydants peut prévenir ou empêcher des anomalies associées au diabète et à ses complications. Plusieurs études ont montré que les régimes supplémentés en vitamines et en minéraux préviennent ou, au moins, atténuent la détérioration organique causée par un stress oxydatif excessif chez les patients diabétiques (Elejalde-Guerra, 2001; Ylonen et al., 2003).

Le métabolisme des lipides et des lipoprotéines est altéré chez les macrosomes La plupart des anomalies lipidiques observées chez les nouveau-nés macrosomiques étaient analogues à celles trouvées chez leurs mères diabétiques. Chez ces bébés, les concentrations sériques en lipides, lipoprotéines et apoB100 étaient plus élevées que celles des bébés issus de mères en bonne santé (Merzouk et al., 2000). Dans le but de comprendre le rôle du métabolisme lipidique dans l'étiopathologie de la macrosomie, nous avons développé dans notre laboratoire un modèle animal. Il a été démontré que l'administration de streptozotocine (STZ), avant la grossesse, affecte la fertilité et altère le développement de l'embryon pendant la période de préimplantation (Vercheval et al., 1990). Pour cette raison, dans notre modèle, nous avons injecté, à des rattes Wistar femelles, par voie intrapéritonéale, une dose unique de $40 \mathrm{mg} / \mathrm{kg}$ de poids corporel de STZ, au $5^{\mathrm{e}}$ jour de la gestation et n'avons observé aucun effet délétère sur le développement de l'embryon. 
Cette dose unique a été choisie après plusieurs essais préliminaires au cours desquels nous avons remarqué que de faibles doses de STZ ne permettaient pas d'obtenir un bon modèle de diabète gestationnel, à cause de la grande variabilité des taux de glucose et de l'absence de macrosomie fœtale. Dans le but d'éviter l'interférence des hormones reproductrices femelles qui sont associées à la prévalence, la susceptibilité et la sévérité de maladies autoimmunes (Buggage et al., 2003), seuls les mâles macrosomiques ont été inclus dans l'étude.

Les rattes gestantes ont été d'excellents modèles expérimentaux parce qu'elles ont montré, au cours de la gestation, une augmentation de VLDL-TAG similaire à celle trouvée chez les humains. La surproduction de VLDL, une caractéristique commune à l'obésité humaine et aux différentes obésités expérimentales, a été une conséquence directe de l'hyperinsulinémie et de l'hyperlipogenèse hépatique (Bioletto et al., 2000). La première observation importante apparente à l'âge de 3 mois, c'est-à-dire à l'âge adulte, est que ces rats avaient pris progressivement du poids et leur gain pondéral a été significativement plus élevé que celui des rats contrôles (Merzouk et al., 2002). En outre, ils ont été hyperglycémiques et hyperinsulinémiques, avec des concentrations élevées en lipide et lipoprotéine. Ces rats obèses ont aussi présenté une augmentation significative des lipides hépatiques, LCAT, LPL, HTGL, et des activités de l'ACAT (acyl Co-A cholesterol acyl transferase), de 1'HMG-CoA (3-hydroxy3-methylglutaryl-CoA) réductase et de la $7 \alpha$ hydroxylase. Pris ensemble, ces résultats suggèrent fortement que ces rats vont développer à l'âge adulte une insulinorésistance.

La macrosomie fœtale retrouvée chez les nouveau-nés de mères GDM, a influencé le métabolisme des lipides et des lipoprotéines à la naissance et surtout à l'âge adulte. La macrosomie observée au cours du diabète gestationnel constitue un important facteur de risque d'obésité à l'âge adulte, de diabète et d'autres complications métaboliques, incluant les anomalies du métabolisme des acides gras essentiels et la dyslipoprotéinémie.

Cytokines et adipokines inflammatoires sont altérées lors du diabète gestationnel et la macrosomie

A travers différents modèles expérimentaux de diabète, il a été bien établi que la sécrétion des cytokines dérivant des cellules $\mathrm{T}$ joue un rôle important dans la régulation du diabète (Rabinovitch, 1994). Sur la base de la production des cytokines, les cellules $\mathrm{T}$ peuvent être classées en deux principales populations, Th1 et Th2. Les cellules Th1 sont responsables de l'immunité à médiation cellulaire et par conséquent favorisent l'inflammation, la cytotoxicité, et retardent l'hypersensibilité alors que les cellules Th2 supportent l'immunité humorale et diminuent les actions inflammatoires des cellules Th1 (Rengarajan et al., 2000). Les cellules Th1 sécrètent IL-2, INF- $\gamma$ et TNF-B tandis que les cellules Th2 sécrètent IL-4, IL5, IL-6, IL-10 et IL-13. Les cytokines Th1, principalement INF- $\gamma$, jouent un rôle pathogénique tandis que les cytokines Th2, principalement IL-4 et IL-10, assurent une fonction régulatrice, et ainsi protègent $\mathrm{du}$ diabète (Wood et al., 1999; Muller et al., 2002). Nous avons aussi étudié l'implication $\mathrm{du}$ système immunitaire dans le diabète gestationnel et la macrosomie. Nous avons montré que, chez les bébés macrosomiques, la différentiation des cellules en Th1/Th2 est orientée vers les cellules Th1 qui sécrètent les cytokines pro-inflammatoires (Atègbo et al., 2006 ; Khan et al., 2006). Nous avons aussi démontré que l'activation et la signalisation calcique des cellules $\mathrm{T}$ sont altérées dans ces deux pathologies (Guermouche et al., 2005; Khan et al., 2006).

A présent, concernant le rôle de 1'IL-6 et de TNF- $\alpha$ dans le diabète gestationnel, nous voudrions mentionner que l'interleukine-6 (IL-6) est secrété aussi bien par les cellules immunocompétentes (comme mentionné cidessus) que par les adipocytes et les macrophages, et sa libération est stimulée par IL-1 et TNF $\alpha$, et inhibée par IL-4 et IL-10 
(cytokines sécrétées par les cellules Th2). L'observation selon laquelle $10-35 \%$ du taux basal corporel d'IL- 6 dérive du tissu adipeux a suscité un intérêt concernant cette cytokine comme étant un possible médiateur des processus métaboliques. Par ailleurs, une corrélation positive a été observée entre 1'IL-6 circulant et l'adiposité (Mohamed-Ali et al., 1997). De plus, une corrélation positive a été trouvée entre la résistance à l'insuline et les taux d'IL-6 circulants (Bastard et al., 2000) qui sont élevés dans le plasma des patients diabétiques de type 2 (Pickup et al., 1997, Muller et al., 2002). Plusieurs études ont démontré que l'obésité est une pathologie qui est médiée par les cytokines proinflammatoires. Dandona et al. (2004) ont établi une co-relation entre l'activation du système immunitaire et le diabète. Plusieurs observations ont aussi indiqué qu'une concentration élevée d'IL-6 prédit le risque de développer l'obésité et un diabète de type 2 (Pickup et al., 1997; Pradhan et al., 2001 ; Vozarova et al., 2001).

Il semble que la résistance à l'insuline, induite par TNF- $\alpha$, serait en partie due à la libération d'IL-6 par les adipocytes. En effet, une récente étude sur 15 sujets a suggéré le rôle de TNF- $\alpha$ (Kirwan et al., 2002) dans cette résistance à l'insuline induite par la grossesse. En outre, une autre étude a démontré une association entre TNF- $\alpha$ et les taux de Cpeptide (Aires et al., 2003). De nos jours, plusieurs études ont montré que les taux de TNF- $\alpha$ devraient être élevés lors du diabète gestationnel (Winkler et al., 2002; Csek et al., 2002).

Depuis qu'il a été montré que les adipocytokines jouent un rôle important dans l'apparition précoce du diabète de type 2 (Greenberg et McDaniel, 2002), le diabète gestationnel représente un modèle idéal pour étudier ces inter-relations. La leptine, une adipocytokine, est aussi produite par le placenta et impliquée dans la régulation du poids et dans le métabolisme lipidique. Kautzky-Willer et al. (2001) ont observé des taux élevés de leptine chez des femmes souffrant du diabète gestationnel. Cependant,
Festa et al. (1999) ont trouvé que le taux de leptine avait diminué lors du diabète gestationnel. En outre, l'adiponcetine et la leptine sont impliquées dans la régulation de l'homéostasie énergétique et l'insulinorésistance. Nous avons récemment montré que les taux d'adipokines (adiponectine et leptine) sont significativement altérés dans une population Tunisienne souffrant du diabète gestationnel et de macrosomie. En conséquence, le diabète gestationnel semble être lié à une augmentation du taux de leptine et des cytokines inflammatoires, tandis que la macrosomie était associée à une chute des taux d'IL-6, de TNF- $\alpha$, de leptine et d'adiponectine (Atègbo et al., 2006).

Ces observations mentionnées cidessus suggèrent que le diabète gestationnel et la macrosomie sont associés à l'altération de la fonction des cellules $\mathrm{T}$ et des adipokines où les cytokines pro-inflammatoires jouent un rôle important dans la pathogenèse de ces deux désordres métaboliques. Par conséquent, une stratégie pour la gestion du diabète gestationnel et de la macrosomie devrait être basée sur l'antagonisme de ces agents.

Les cellules-T présentent un défaut dans la signalisation cellulaire au cours du diabète gestationnel et la macrosomie

Au cours de l'activation des cellules-T, l'un des évènements précoces déclenché suite à l'hydrolyse du phosphatidyl-inositoldiphosphate et catalysé par la phospholipase $\mathrm{C}$ (PLC) a été l'augmentation de la concentration en calcium intracellulaire libre, $\left[\mathrm{Ca}^{2+}\right]$ i. En outre, la PLC a augmenté l'inositol triphosphate qui mobilise le calcium des réserves endoplasmiques et le diacylglycérol qui active la protéine kinase C. Selon le modèle capacitatif de l'entrée du calcium, on admet qu'il est d'abord libéré du réticulum endoplasmique (ER) par l'activation des récepteurs des cellules-T (TCR) et puis expulsé dans le milieu extracellulaire; en retour, ces cellules remplissent leur réserve intracellulaire en ouvrant les canaux calciques (Putney, 1997). L'ionomycine, à $50 \mathrm{nM}$, ouvre les canaux calciques et la thapsigargine 
(TG) recrute le calcium intracellulaire libre des réserves du réticulum endoplasmique. Nous avons observé que l'ionomycine induit une augmentation plus importante du calcium intracellulaire libre dans les cellules-T de rattes GDM et de leurs nouveau-nés obèses que chez les contrôles. Avec un milieu calcique à $100 \%$ et à $0 \%$, la TG induit une augmentation plus importante du calcium intracellulaire libre dans les cellules-T des rattes GDM et de leurs bébés macrosomiques que des animaux témoins (Guermouche et al., 2004). Ces résultats démontrent que la signalisation calcique des cellules-T est altérée au cours du diabète gestationnel et la macrosomie.

\section{Les cellules- $T$ régulatrices (T-Reg) sont surexprimées au cours du diabète gestationnel}

Il a été suggéré qu'au cours de leur développement dans le thymus, les cellules-T auto-réactives sont supprimées par un processus appelé "tolérance centrale". Cependant, cette sélection négative étant incomplète, celles qui échappent à cette suppression clonale sont supposées être contrôlées à la périphérie par les cellules-T régulatrices (T-Reg) qui suppriment l'activation et l'expansion des cellules autoréactives évadées (Fontenot et al., 2003). Ainsi, les cellules T-Reg contrôlent la délicate balance entre immunité et tolérance, ce qui explique leur important rôle lors des maladies autoimmunes, du cancer, de la tolérance à la transplantation et de l'allergie. Cependant, leur implication dans le diabète gestationnel et la macrosomie n'est pas bien connue. Ces cellules expriment constitutivement $\mathrm{CD} 4^{+} \mathrm{CD} 25^{+}$, un facteur de transcription appelé Foxp3 et CTLA-4. Holm et al. (2006) ont rapporté que la fréquence des cellules-T $\mathrm{CD} 4^{+} \mathrm{CD} 25^{+}$était significativement plus élevée chez les enfants nés de mères diabétiques de type 1 que chez ceux nés de mères normales. En outre, chez ces enfants, ces cellules présentent une mémoire plus prononcée du phénotype avec une augmentation de l'expression de CCR4 et une diminution de CD62L, suggérant une activation précoce du système immunitaire fœtal, conséquence du statut immunitaire maternel.

\section{L'Acide Docosahexaenoïque (DHA) exerce des effets bénéfiques sur le diabète gestationnel et la macrosomie}

Les acides gras polyinsaturés en n-6 (PUFAs), en particulier l'acide arachidonique, via la production de prostaglandines et de leucotriènes induisent des effets proinflammatoires. D'autre part, il a été montré que les PUFAs en n-3 étaient des agents antiinflammatoires et immunosuppresseurs. Khan et Merzouk (2003) puis Yessoufou et al., (2006) ont récemment démontré qu'un régime riche en PUFAs n-3 (acide eicosapentaenoïque et docosahexaenoïque), donné à des bébés obèses, nés de rattes diabétiques, a exercé un effet bénéfique : ce régime a significativement diminué l'incidence de la macrosomie, corrigé les anomalies du métabolisme lipidique et restauré le statut antioxydant altéré chez ces animaux. De plus, ce régime avait augmenté la différentiation des cellules Th0 en un phénotype Th2 (effet protecteur) chez ces bébés obèses (Khan et al., 2006).

La programmation in utero semble être responsable des modifications observées à l'âge adulte

David Barker (1995) écrivait : “'L'hypothèse des origines fotales énonce que la malnutrition fœtale, en milieu ou à une période tardive de la gestation, qui aboutit à une croissance fœtale disproportionnée, programme plus tard une maladie coronarienne', Le processus, par lequel un stimulus ou une lésion à une période délicate ou critique du développement entraîne des effets à long terme, est appelé programmation. En fait, cette programmation in utero semble créer une sorte de "mémoire métabolique" puisque les anomalies observées dans le système physiologique pendant la période gestationnelle sont responsables, à l'âge adulte, de "l'apparition" de maladies comme 
le diabète de type 2 et l'obésité, associées au syndrome métabolique. Il existe, chez la mère et le fœtus âgé de 6 mois, une bonne corrélation entre les taux plasmatiques en cholestérol et, certaines altérations telles que les taux élevés de triacylglycérol (TAG), apoB100, VLDL, LDL ont souvent persisté chez les nouveau-nés macrosomiques après un mois de vie (Merzouk et al., 2002). Nous avons montré, chez les rats macrosomiques nés de mères GDM, que la plupart des paramètres lipidiques se sont normalisés vers l'âge de 2 mois et ont réapparu à l'âge adulte. Ces observations suggèrent qu'il existe une sorte de "mémoire métabolique" in utero qui est, en quelque sorte, dormant pendant l'enfance et qui induirait, chez ces animaux à l'âge adulte, une augmentation des taux de glucose, d'insuline et de lipides (Merzouk et al., 2003).

Les conséquences du diabète gestationnel ne se limitent pas au risque de malformations congénitales et de macrosomie fœtale. Il a été également démontré qu'indépendamment de leur poids à la naissance, les enfants ou jeunes adultes de mères diabétiques ont présenté, quel que soit le type de diabète maternel, une plus grande fréquence d'insulino-résistance et d'intolérance au glucose que les enfants nés de mères non diabétiques (Sobngwi et al., 2003). Ce phénomène ne peut s'expliquer entièrement par la transmission de gènes prédisposant au diabète mais il traduit un effet persistant de perturbations de la croissance et du métabolisme induites par l'environnement intra-utérin diabétique. Chez l'animal, des manipulations expérimentales des conditions de gestation ont apporté de solides arguments en faveur du rôle causal des conditions nutritionnelles intra-utérines sur la santé ultérieure (Armitage et al., 2005). Les mécanismes précis de ces relations ne sont pas bien connus mais certaines hypothèses ont été avancées: des conditions de grossesse particulières présenteraient une entrave au développement de certaines lignées cellulaires ou, au contraire, favoriseraient le développement préférentiel d'autres lignées. Il est également possible que des altérations fonctionnelles soient en cause telles que, par exemple, des modifications durables de l'expression de certains gènes. La progression dans la connaissance des mécanismes épigénétiques offrirait un cadre conceptuel pour explorer les relations entre environnement intra-utérin au début de la vie et santé à long terme.

Les modifications métaboliques in utero, responsables de l'apparition de ces anomalies physiologiques chez l'adulte, restent à être approfondies. Cependant, il est possible que l'hyperinsulinémie fœtale soit un facteur tératogène endogène au cours des périodes critiques de développement du fœetus, aboutissant à des changements structuraux permanents et fonctionnels des organes et une programmation conséquente de la "mémoire métabolique"' (Dömer et Plagemann, 1994). La mauvaise régulation des récepteurs à l'insuline chez les fotus hyperinsulinémiques pourrait augmenter ses sites de liaison et, ainsi, ses effets métaboliques.

Des études expérimentales réalisées sur des rattes ont montré le lien étroit entre l'environnement nutritionnel utérin au cours de la vie fotale et le risque à développer des maladies dégénératives à l'âge adulte. Erhuma et al. (2007) ont nourri, à différentes périodes de la gestation, des rattes gestantes avec un régime contrôle et un régime carencé en protéine; les nouveau-nés ont été étudiés à différents moments de la croissance, soit à 1 , 9 et 18 mois. Tous les rats âgés de 1 et 9 mois, issus de mères exposées au régime test, avaient des concentrations plasmatiques en triglycérides, cholestérol, glucose et insuline semblables à celles des contrôles; à 18 mois, il est apparu l'évidence d'une hypertriglycéridémie liée au faible régime protéique et une insulino-résistance. En plus, à cet âge, ils ont présenté une stéatose hépatique et avaient 2 à 3 fois plus de triglycéride que les animaux contrôles. Ces changements phénotypiques étaient accompagnés par des modifications dans l'expression des ARNm et des facteurs de transcription comme SREBP1c et ChREBP. Le rôle de la SREBP-1c 
(sterol-regulatory-element-binding protein1c) dans la régulation du métabolisme hépatique est maintenant bien établi. En effet, l'insuline et le glucose stimulent la synthèse de la SREBP-1c qui induit, dans le foie et le tissu adipeux, l'expression des gènes impliqués dans la lipogenèse, surtout la synthèse des acides gras et des triacylglycérols (TAG) (Edwards et al., 2000).

Les résultats obtenus par Erhuma et al. (2007) ont indiqué que la restriction protéique prénatale programmait le développement d'un syndrome métabolique qui apparaît seulement à l'âge adulte, avec implication de l'expression altérée des médiateurs clés du phénotype programmé comme SREBP-1c et ChREBP, mais les bases du changement du statut métabolique qui survient entre 9 et 18 mois demeurent, à nos jours, non identifiées. Ces résultats sont cependant en accord avec ceux de Reusens et Remacle (2005) qui ont observé qu'à certaines étapes critiques du développement embryonnaire, un déséquilibre nutritionnel chez la mère ou des altérations du transport placentaire des nutriments, menant à une malnutrition fotale, pourraient déclencher, chez le fœtus, des adaptations métaboliques ainsi qu'une redistribution des ressources vers les organes nobles comme le cerveau, au détriment d'autres organes tels que les viscères. Ces adaptations et ces redistributions des ressources pourraient alors entraîner des altérations dans la croissance et les fonctions de ces organes et mener à terme au syndrome métabolique. On conçoit alors que le déficit nutritionnel global, en tant que réduction de l'apport calorique, peut perturber l'élaboration de l'individu.

Des études expérimentales ont mis en évidence le rôle clé de l'hypothalamus fotal qui peut être "programmé", par des changements transitoires de l'état endocrine prénatal. Nous pouvons citer les travaux de Yura et al. (2005) qui ont montré que la malnutrition in utero est étroitement associée à l'obésité à l'âge l'adulte. Ils ont aussi démontré que la leptine précoce modifiait, chez des souris sous-alimentées, la régulation énergétique par l'hypothalamus et contribuait au "développement des origines de l'obésité',. Toutefois, l'implication d'autres facteurs dans les modifications in utero reste à être explorée dans le futur. Il a été également démontré que les perturbations observées chez les bébés issus de rattes rendues diabétiques sont accompagnées par, et souvent corrélées avec, des malformations périnatales acquises des noyaux hypothalamiques ventromédians et particulièrement des altérations des neurones hypothalamiques exprimant des neuropeptides orexigéniques comme le neuropeptide Y dans le noyau hypothalamique arqué (Plagemann et al., 1999). Ces perturbations hypothalamiques peuvent être co-responsables d'altérations tardives de la prise alimentaire, du poids corporel et du métabolisme glucidique chez les nouveau-nés issus de mères diabétiques non traitées. Franke et al. (2005) ont montré, chez des rattes, que le diabète gestationnel non traité a entraîné de graves conséquences pour la différentiation des neurones hypothalamiques du nouveau-né. Ces altérations neuropeptidergiques hypothalamiques sont évitables par la normalisation de l'hyperglycémie gestationnelle. L'augmentation du neuropeptide Y chez les nouveau-nés issus de rattes hyperglycémiques s'explique par une "mauvaise programmation" du système neuropeptidergique due à une exposition au diabète gestationnel non traité. Ces altérations acquises observées chez les bébés pourraient fortement contribuer à augmenter le risque à développer l'hyperphagie, le surpoids et des perturbations diabétogéniques à l'âge adulte. L'organisation altérée des systèmes de régulation neuropeptidergiques peut, en terme de prédisposition, aboutir à un dysfonctionnement ultérieur ou, plus tard, à une altération des neurones hypothalamiques à travers une glucotoxicité et une insulino-résistance hypothalamique progressive.

\section{Conclusion}

La macrosomie fœtale est un facteur de risque pour l'obésité ultérieure, le diabète et la 
dyslipoprotéinémie. La macrosomie est une pathologie où le développement in utero, dans un contexte d'hyperglycémie et d'hyperlipidémie, joue un rôle important dans la programmation de la "mémoire métabolique". Les causes primaires de l'insulino-résistance au cours du diabète de type 2 et du syndrome métabolique devraient être recherchées en dehors des cellules $\beta$ pancréatiques, dans le réseau complexe de neurones, d'hormones et de signaux métaboliques qui orchestrent le métabolisme des tissus. La macrosomie est une pathologie multifactorielle, impliquant les anomalies des lipides, des lipoprotéines, du statut antioxydant, et l'activation du système immunitaire (prédisposition au phénotype Th1). Le phénotype des cellules-T régulatrices (T-Reg) est surexprimé chez les mères GDM et leurs enfants macrosomiques. Cependant, il reste à déterminer comment la série des modulateurs métaboliques tels que l'insuline et les lipides influencerait l'activation des cellules-T au cours de ces pathologies.

\section{REMERCIEMENTS}

Les auteurs remercient très sincèrement le Professeur Naim A. KHAN, Département de Physiologie, Faculté des Sciences de la Vie, Université de Bourgogne, UPRES EA 4183 "Lipides et Signalisation Cellulaires", Dijon France pour son assistance et sa contribution.

\section{REFERENCES BIBLIOGRAPHIQUES}

Aires V, Adote S, Hichami A, Moutairou K, Boustani E, Khan NA. 2003. Modulation of intracellular calcium concentrations and T-cell activation by prickly pear polyphenols. Mol. Cell. Biochem., 260: 103-110.

Armitage JA, Taylor PD, Poston L. 2005. Experimental models of developmental programming: consequences of exposure to an energy rich diet during development. J. Physiol., 565(Pt 1): 3-8.

Atègbo JM, Grissa O, Yessoufou A, Hichami A, Dramane KL, Moutairou K, Miled A, Grissa A, Jerbi M, Tabka Z, Khan NA.
2006. Modulation of adipokines and cytokines in gestational diabetes and macrosomia. J. Clin. Endocrinol. Metab., 91: 4137-4143.

Barker DJ. 1995. Fetal origins of coronary heart disease. BMJ., 311: 171-174.

Basdevant A, Laville M, Ziegler O. 1998. [Practice guideline for the diagnosis, prevention, treatment of obesity in France. Groupe de Travail charge de la mise au point des "Recommandations pour le diagnostic, la prévention et le traitement des Obésités en France"]. Diabetes Metab., 24: 10-42.

Bastard JP, Maachi M, Van Nhieu JT, Jardel C, Bruckert E, Grimaldi A, Robert JJ, Capeau J, Hainque B. 2000. J. Clin. Endocrinol. Metab., 87: 2084-2089.

Bioletto S, Golay A, Munger R, Kalix B, James RW. 2000. Acute hyperinsulinemia and very-low-density and low-density lipoprotein subfractions in obese subjects. Am. J. Clin. Nutr., 71: 443-449.

Buggage RR, Matteson DM, Shen DF, Sun B, Tuaillon N, Chan CC. 2003. Effect of sex hormones on experimental autoimmune uveoretinitis (EAU). Immunol. Invest.,.32(4): 259-273.

Coustan DR. 1997. Gestational diabetes: a continuum of risk. Eur. J. Endocrinol., 137: 13-14.

Cox NJ. 1994. Maternal component in NIDDM transmission. How large an effect? Diabetes, 43: 166-168.

Cseh K, Baranyi E, Melczer Z, Csakany GM, Speer G, Kovacs M, Gero G, Karadi I, Winkler G. 2002. The pathophysiological influence of leptin and the tumor necrosis factor system on maternal insulin resistance: negative correlation with anthropometric parameters of neonates in gestational diabetes.Gynecol Endocrinol., 16: $453-460$.

Dandona P, Aljada A, Bandyopadhyay A. 2004. Inflammation: the link between insulin resistance, obesity and diabetes. Trends Immunol., 25:4-7.

Dörner G, Plagemann A. 1994. Perinatal hyperinsulinism as possible predisposing 
factor for diabetes mellitus, obesity and enhanced cardiovascular risk in later life. Horm. Metab. Res., 26(5): 213-221. Review.

Edwards PA, Tabor D, Kast HR, Venkateswaran A. 2000. Regulation of gene expression by SREBP and SCAP. Biochim. Biophys. Acta., 1529: 103-113.

Elejalde Guerra JL. 2001. Oxidative stress, diseases and antioxidant treatment. Ann. Med. Intern., 18: 326-335.

Erhuma A, Salter AM, Sculley DV, LangleyEvans SC, Bennett AJ. 2007. Prenatal exposure to a low-protein diet programs disordered regulation of lipid metabolism in the aging rat. Am. J. Physiol. Endocrinol. Metab., 292(6): E1702-1714.

Evers IM, de Valk HW, Mol BW, ter Braak EW, Visser GH. 2002. Macrosomia despite good glycaemic control in Type I diabetic pregnancy; results of a nationwide study in The Netherlands. Diabetologia 45:1484-489.

Festa A, Shnawa N, Krugluger W, Hopmeier P, Schernthaner G, Haffner SM. 1999. Relative hypoleptinaemia in women with mild gestational diabetes mellitus. Diabet. Med., 16: 656-662.

Fontenot JD, Gavin MA, Rudensky AY. 2003. Foxp3 programs the development and function of $\mathrm{CD} 4+\mathrm{CD} 25+$ regulatory $\mathrm{T}$ cells. Nat. Immunol., 4(4): 330-336.

Franke K, Harder T, Aerts L, Melchior K, Fahrenkrog S, Rodekamp E, Ziska T, Van Assche FA, Dudenhausen JW, Plagemann A. 2005. 'Programming' of orexigenic and anorexigenic hypothalamic neurons in offspring of treated and untreated diabetic mother rats. Brain Res.; 1031(2): 276283.

Gabbe S. 1986. Gestational Diabetes Mellitus. N. Engl. J. Med., 315: 10251026.

Guermouche B, Yessoufou A, Soulimane N, Merzouk H, Moutairou K, Hichami A, Khan N. 2004. N-3 fatty acids modulate $\mathrm{T}$-cell calcium signaling in obese macrosomic rats. Obes Res., 12: 17441753.
Greenberg AS, McDaniel ML. 2002. Identifying the links between obesity, insulin resistance and beta-cell function: potential role of adipocyte-derived cytokines in the pathogenesis of type 2 diabetes Eur. J. Clin. Invest., 32: 24-34.

Guemouri L, Artur Y, Herbeth B. 1991. Biological variability of superoxide dismutase, glutathione peroxidase, and catalase in blood. Clin. Chem., 37: 19321937.

Holm BC, Svensson J, Akesson C, Arvastsson J, Ljungberg J, Lynch K, Ivarsson SA, Lernmark A, Cilio CM. 2006. Evidence for immunological priming and increased frequency of CD4+ CD25+ cord blood T cells in children born to mothers with type 1 diabetes. Clin Exp Immunol., 146(3): 493-502.

Hunt JV, Smith CCT, Wolf SP. 1990. Autoxidative glycosylation and possible involvement of peroxides and free radicals in LDL modification by glucose. Diabetes, 39: 1420-1424.

Jensen DM, Damm P, Sorensen B, MolstedPedersen L, Westergaard JG, Korsholm L, Ovesen P, Beck-Nielsen H. 2003. Proposed diagnostic thresholds for gestational diabetes mellitus according to a 75-g oral glucose tolerance test. Maternal and perinatal outcomes in 3260 Danish women. Diabet. Med., 20: 51-57.

Kamath U, Rao G, Raghothama C, Rai L, Rao P. 1998. Erythrocyte indicators of oxidative stress in gestational diabetes Acta Paediatr., 87: 676-679.

Kautzky-Willer A, Pacini G, Tura A, Bieglmayer C, Schneider B, Ludvik B, Prager R, Waldhausl W. 2001. Increased plasma leptin in gestational diabetes. Diabetologia, 44: 164-172.

Khan NA, Yessoufou A, Kim M, Hichami A. 2006. N-3 fatty acids modulate Th1 and Th2 dichotomy in diabetic pregnancy and macrosomia. J. Autoimmun., 26: 268-277.

Kirwan JP, Hauguel-De Mouzon S, Lepercq J, Challier JC, Huston-Presley L, Friedman JE, Kalhan SC, Catalano PM. 2002. TNFalpha is a predictor of insulin resistance 
in human pregnancy. Diabetes, 51: 22072213.

Merzouk H, Madani S, Korso N, Bouchenak M, Prost J, Belleville J. 2000. Maternal and fetal serum lipid and lipoprotein concentrations and compositions in type 1 diabetic pregnancy: relationship with maternal glycemic control. J. Lab. Clin. Med., 136: 441-448.

Merzouk H, Madani S, Hichami A, Prost J, Belleville J, Khan NA. 2002. Age-related changes in fatty acids in obese offspring of streptozotocin-induced diabetic rats. Obes. Res., 10: 703-714.

Merzouk S, Hichami A, Madani S, Merzouk H, Berrouiguet AY, Prost J, Moutairou K, Chabane-Sari N, Khan NA. 2003. Antioxidant status and levels of different vitamins determined by high performance liquid chromatography in diabetic subjects with multiple complications. Gen. Physiol. Biophys., 22: 15-27.

Merzouk H, Khan NA. 2003. Implication of lipids in macrosomia of diabetic pregnancy: can $n-3$ polyunsaturated fatty acids exert beneficial effects? Clin Sci (Lond): 105(5): 519-529.

Mohamed-Ali V, Goodrick S, Rawesh A, Katz DR, Miles JM, Yudkin JS, Klein S, Coppack SW. 1997. Subcutaneous adipose tissue releases interleukin- 6 , but not tumor necrosis factor-alpha, in vivo. J. Clin. Endocrinol. Metab. 82: 41964200.

Muller S, Martin S, Koenig W, HanifiMoghaddam P, Rathmann W, Haastert B, Giani G, Illig T, Thorand B, Kolb H. 2002. Impaired glucose tolerance is associated with increased serum concentrations of interleukin 6 and coregulated acute-phase proteins but not TNF-alpha or its receptors. Diabetologia, 45: 805-812.

Ornoy A, Zaken V, Kohen R. 1999. Role of reactive oxygen species (ROS) in the diabetes-induced anomalies in rat embryos in vitro: reduction in antioxidant enzymes and low-molecular-weight antioxidants (LMWA) may be the causative factor for increased anomalies. Teratology, 60: 376-386.

Pickup JC, Mattock MB, Chusney GD, Bart D. 1997. NIDDM as a disease of the innate immune system: association of acute-phase reactants and interleukin-6 with metabolic syndrome $\mathrm{X}$ Diabetologia, 40: 1286-1292.

Plagemann A, Harder T, Melchior K, Rake A, Rohde W, Dörner G. 1999. Elevation of hypothalamic neuropeptide Y-neurons in adult offspring of diabetic mother rats. Neuroreport., 10(15): 3211-3216.

Pradhan AD, Manson JE, Rifai N, Buring JE, Ridker PM. 2001. C-reactive protein, interleukin 6, and risk of developing type 2 diabetes mellitus. JAMA 286: 327-334.

Rabinovitch A. 1994. Immunoregulatory and cytokine imbalances in the pathogenesis of IDDM. Therapeutic intervention by immunostimulation? Diabetes, 43: 613621.

Rengarajan J, Szabo SJ, Glimcher LH. 2000. Transcriptional regulation of Th1/Th2 polarization. Immunol. Today, 21: 479483.

Reusens B, Remacle C. 2005. Development of diabetes, experimental models. Journ Annu Diabetol Hotel Dieu.; Médecine_Sciences Flammarion, 77-84.

Sobngwi E, Boudou P, Mauvais-Jarvis F, Leblanc H, Velho G, Vexiau P, Porcher R, Hadjadj S, Pratley R, Tataranni PA, Calvo F, Gautier JF. 2003. Effect of a diabetic environment in utero on predisposition to type 2 diabetes. Lancet.; 361(9372): 1861-1865.

Soulaimann-Mokhtari NA, Guermouche B, Yessoufou A, Saker M, Moutairou K, Hichami A, Surkan PJ, Hsieh CC, Johansson AL, Dickman PW, Cnattingius S. 2005. Modulation of lipid metabolism by n-3 polyunsaturated fatty acids in gestational diabetic rats and their macrosomic offspring.. Obstet. Gynecol., 104: 720-726.

Tauber M, Jouret B, Fouroux M, Rochiccioli P. 1998. Management of obesity in children and adolescents requires 
mobilization of all specialists. Arch. Pédiatr, 5: 595-598.

Therond P, Bonnefont-Rousselot D, DavitSpraul A, Conti M, Legrand A. 2000. Biomarkers of oxidative stress: an analytical approach. Curr. Pin. Clin. Nut. Metab. Care, 3: 373-384.

Vercheval M, De Hertogh R, Pampfer S, Vanderheyden I, Michiels B, De Bernardi P, De Meyer R. 1990. Experimental diabetes impairs rat embryo development during the preimplantation period. Diabetologia, 33(4): 187-1891.

Vozarova B, Weyer C, Hanson K, Tataranni PA, Bogardus C, Pratley RE. 2001. Circulating interleukin-6 in relation to adiposity, insulin action, and insulin secretion. Obes. Res., 9: 414-417.

Winkler G, Cseh K, Baranyi E, Melczer Z, Speer G, Hajos P, Salamon F, Turi Z, Kovacs M, Vargha P, Karadi I. 2002. Tumor necrosis factor system in insulin resistance in gestational diabetes. Diabetes Re.s Clin. Pract., 56: 93-99.
Wood SC, Rao TD, Frey AB. 1999. Multidose streptozotocin induction of diabetes in $\mathrm{BALB} / \mathrm{cBy}$ mice induces a $\mathrm{T}$ cell proliferation defect in thymocytes which is reversible by interleukin-4. Cell Immunol ., 192: 1-12.

Yessoufou A, Soulaimann N, Merzouk S, Moutairou K, Ahissou H, Prost J, Simonin AM, Hichami A and Khan NA. 2006. N-3 fatty acids modulate antioxidant status in diabetic rats and their macrosomic offspring. Int J Obes., 30:739-750.

Ylonen K, Alfthan G, Groop L, Saloranta C, Aro A, Virtanen SM. 2003. Dietary intakes and plasma concentrations of carotenoids and tocopherols in relation to glucose metabolism in subjects at high risk of type 2 diabetes: the Botnia Dietary Study. Am. J. Clin. Nutr., 77: 1434-1441.

Yura S, Itoh H, Sagawa N, Yamamoto $\mathrm{H}$, Masuzaki H, Nakao K, Kawamura M, Takemura M, Kakui K, Ogawa Y, Fujii S. 2005. Role of premature leptin surge in obesity resulting from intrauterine undernutrition. Cell Metab., 1: 371-378. 